\title{
Grounding Grids Corrosion Diagnosis Based on A Block Dividing Approach
}

\author{
Ni Yunfeng \\ School of Electrical Engineering, Xi'an University of \\ Science \& Technology Xi'an, 710054 \\ Xi'an ,China \\ $\mathrm{Xu} \mathrm{Li}$ \\ School of Electrical Engineering, Xi'an University of \\ Science \& Technology, Xi'an, 710054 \\ Xi'an ,China \\ e-mail: niyunfeng@xust.edu.cn
}

\author{
Wang Shuqi \\ School of Electrical Engineering, Xi'an University of \\ Science \& Technology , Xi'an, 710054 \\ Xi'an,China \\ e-mail: wangshuqi@xust.edu.cn
}

\begin{abstract}
To benefit the corrosion diagnosis, the concepts of blocks and their prototypes, uncertain-branch groups and their prototypes, projection of blocks and part of touchable nodes are defined. A new approach of grounding grids corrosion diagnosis by blocks is putting forward. According to the desired corrosion diagnosis area, the blocks together with their projections and the parts of touchable nodes, which participate in diagnosis are determined. According to the testing data of the corresponding parts, the resistances of intrinsic branches in a block can be determined by a linear least square method, based on which, the resistances of clear branches in the block can be worked out. The ranges of resistances of uncertain-branches in the block are also evaluated by a dichotomy algorithm. With the proposed approach, a grounding grid can be divided into several small scaled sub-grids to solve. As a result, the efficiency is improved and the cross-interference is reduced. An experimental grounding grid with sixty branches is used as an example. The results of corrosion diagnosis show that the proposed approaches are feasible and only a few tests are needed to meet the requirement of diagnosing a certain part of the grounding grid.
\end{abstract}

Keywords-grounding grids; corrosion diagnosis; diagnosis within a block; test with a part; dichotomy algorithm

\section{INTRODUCTION}

Grounding grid is an important facility to ensure the electrical equipment and personal safety, and analysis and optimization designs of grounding grid have been studied in numerous literature [1-2]. Generally made of flat steels, grounding grids are easily corroded after long time operation which results in the demand of corrosion diagnosis and repair measures.

Two main categories of grounding grids corrosion diagnosis can be distinguished : electromagnetic field approach based and circuit analysis method based.

The former is typically represented in [3] and [4], which introduce electromagnetic field approach to locate the broken branches in grounding grids conductors

As for the second method, it is a typical kind of analogous circuit corrosion diagnosis means [5-7]. In [8] resistance of each conductor in grids is calculated by resistance measurement based on Tellegren's theorem.
Establishing node voltage equations and using simplex method,we solve the resistance increment of each conductor [9-11]. Tabu search algorithm is prompted in solving node voltage equations[12]. With multiple measurements of each excitation section,the optimal solution in least square method is obtained by gaining the number of equation as well as adopting iterative method [13]Software system is also developed in grounding grids corrosion diagnosis[14].

Hierarchical reduction algorithm is suggested to analyze the possibility of grounding grids and distinguish clear branches from uncertain-branches. On this basis, it puts forward a thought of ascent layer-by-layer which can be used in corrosion diagnosis [15].

Based on complete test of the grounding grids, the existing method [5-15] diagnose each branch of grids. We can also separate grids into several parts ,however, every part is a large scale, especially when the number of knickpoints in breaking parts is large. So we have to build equivalent network between branches within knickpoints.

To large network corrosion diagnosis, not only do we need complex matrix calculation ,but also measurement error cross-interference exists .To make matters worse, when there is a larger measurement error data, the effect on the diagnosis of all the branches would be significant. Meanwhile, in some cases, diagnosing some parts of grids or rechecking the result of diagnosis, the disadvantage of the methods above would be obvious.

Moreover, when we solve intrinsic ground grids, nonlinear iteration process is still needed [15].

In light of the correlation between branches, a block model is presented in this paper. The method tends to separate large grids into several independent parts, based on which, a new grounding grids corrosion diagnosis by blocks is proposed. With the feasible approach, computational efficiency is improved and the effect of error cross-interference is decreased. What's more ,nonlinear iterative process is successfully avoided in the process of solving intrinsic branches. 


\section{THE BLOCKS MODEL OF GROUNDING GRID}

\section{A. Research basis Selecting a Template}

Reference [15] maximally splits grounding grids into several quasi element blocks bounded by touchable nodes. On the premise of retaining all the touchable nodes in quasi element plate, a maximum of series and parallel branches are merged into element blocks. According to circuit net theory, untouchable nodes are stripped in order to get corresponding unit network. Then touchable grounding grids which only possess touchable nodes are obtained by synthesizing all the element network. Combing parallel branches of touchable grounding grids, intrinsic grounding grid is gained which has only a branch(called intrinsic branch) at most .Quasi element block, element block and unit network are separately called actual grounding grids, element block network and touchable grounding grid at three levels.

The paper puts forward the block model of grounding grids.

\section{B. Quasi block and its autonomous and non-autonomous parts}

To divide large-scale grounding grids into small network based on the correlation of each branch of grids, we need to define the concept of block and its prototypes.

If all the branches in a unit network are clear, and the resistance of these branches can be solved without the need of other branch resistance diagnosis results, then the unit which the network is in is an autonomous unit.

If there is at least one unknown branch in a network in which the resistance solving needs the diagnosis of other units, we define this unit as non-autonomous unit.

If there are parallel branches(whether these branches are clear or not) between two units, the two units are interconnecting with each other, so are the elements and branches in the two units.

In the grounding grids, the set that are composed of associated branches is called a quasi block.

The corresponding branches set of an autonomous unit in a quasi block is called an autonomous part. The network that is composed of interconnected nonautonomous units and autonomous units associated with is meant by a non-autonomous part.

From what has been discovered above, units of a nonautonomous part are interrelated, but they are independent from other non-autonomous units.

\section{Blocks and their prototypes}

A block is a subset of a quasi block. It is the minimum set of all the branch resistance or the range of value that depends on branch resistance in each net involved. As a result, branch resistance in each block can be solved respectively. Blocks may be different as the divided branches are different. blocks.

Some blocks in a quasi block may own some public

The principal of division is as follows:

1) If we need to solve a branch that fells on an autonomous part of a quasi block, then each part of the autonomous unit in this branch makes up a block which is called autonomous block, and its prototype is unit network.

2) If there is at least one branch that needs to be solved fells on a non-autonomous part of a quasi block, then the each part of the non-autonomous unit in this branch form a block which is called non-autonomous block, and its original prototype is a network that is composed of nonautonomous units in non-autonomous parts as well as the network which is combined by parallel branches(all these branches are intrinsic branches ) of the unit network in autonomous units. If some branches that need solved do not locate in non-autonomous blocks, then each automatic part containing these branches forms automatic block respectively, and its prototype is element network corresponding automatic unit.

It is thus clear that the branch in the prototype is clear and its resistance can be uniquely determined.

A set of intrinsic branches corresponding to a block is called the projection on its intrinsic grounding grids.

A set of intrinsic branches, and their public point is a touchable node, is called a division of the node.

In a block if all the girds branches are clear, then the block is a clear block.

If all branches of an element block are clear but level branches corresponding to grounding grids are not clear, then the block is an quasi clear block.

In a block, if all the girds branches of element blocks are not clear, then we call the block an uncertain block.

If there are both clear branches and uncertain branches in a block, then we call the block a mixed block.

D. Uncertain branches and their prototypes

If there are parallel branches which are uncertain between two element networks, then they are intercrossing.

For a set composed of several element networks, if element networks involved are inter-crossing and there is no network outside inter-crossing the element network inside, then the set is an element network group. If an element network inter-crosses no other network, then the network itself forms an element network group.

In a grounding grid, if branches of an element block in an element network group are uncertain, then their corresponding branches in the grounding grids form a uncertain branch group, we call uncertain branch group I.

If both uncertain branches and clear branches exist in a element network group, we retain clear branches and replace uncertain branches with their corresponding ones in grounding grids, then the subnet work so obtained is called uncertain branch group III.

The prototypes of uncertain branch group I and III are the result of combining parallel branches of relevant element network. The branches in prototypes are all clear and their resistance can be uniquely determined.

If a clear branch of a element block corresponds to a group of uncertain branches in grounding grids, then we consider that those uncertain branches consist of uncertain branch group II and its prototype is clear branch in the element block and their resistance can be also uniquely determined.

Accordingly, the branches in prototypes of uncertain branch group are all clear and their resistance can be 
uniquely determined. What's more, quasi clear block can only contain the uncertain branch group II, uncertain block can only compose the uncertain branch group I, mix block can only contain uncertain branch group I III.

\section{CORROSSION DIAGNOSIS BASED ON A BLOCK DIVIDING APPROACH}

On the basis of the blocks model of grounding grids, a new approach of grounding grids corrosion diagnosis by blocks is putting forward in this section. The main steps included are as follows:

1) In light of requirements (such as the rang of corrosion diagnosis or the rang of test data that need to review), we shall determine blocks involved in diagnosis and base on these blocks to ascertain the projection of partition involved.

2) For the public nodes in each partition we make test, and test date is used to solve the intrinsic branch set.

3) In view of the solution of intrinsic branch set, we solve the resistance of clear branches that selected from grounding grids by blocks and confirm the rang of resistance in uncertain branches.

\section{A. Linear method to solve the resistance of intrinsic branches}

For the touchable node $i$, suppose that a set named $\beta$ of other nodes in the part, under the drive of constant current source and according to KCL, we will get

$$
I_{i}=\sum_{j \in \beta} V_{j, i} G_{i, j}
$$

Where $V_{i, j}$ represents the voltage between touchable node $i$ and $j . G_{i, j}$ is the conductance of intrinsic branch between node $i$ and $j, I_{i}$ is injection current from node $i$.

Hence, in a given excitation location, when the voltage measurement data of node $i$ as well as its adjacent nodes is obtained can we build a linear equation of conductance in intrinsic branches relative to the partition of node $i$.

If the number of intrinsic branches which possess node $i$ as their tip node is $\mathrm{M}$, then the number of adjacent nodes of $i$ is also $\mathrm{M}$. Therefore, we need to select $\mathrm{M}$ independent excitation locations. During each excitation we measure the voltage between node $i$ and its adjacent nodes. From (1), we can get $\mathrm{M}$ independent equations.

The test above is node $i$ oriented test, that is to say, equations which are node $i$ oriented are node $i$ oriented equations. They can be expressed in matrix below.

$$
I=V G
$$

As is indicated in (2), the conductance of intrinsic branches which are adjacent to node $i$ can be determined by a linear least square method and nonlinear iterative process is avoided. Based on which, the resistance of each intrinsic branch can be worked out. For the intrinsic branch $i$, there are

$$
R_{i}=1 / G_{i}
$$

B. Resistance corrosion diagnosis of grounding grids branches based on a block diving approach

\section{1) Main process}

Based on the set of intrinsic branches, the resistance of grounding grids branches can be tested using corrosion diagnosis by blocks. The flow chart of diagnosis for a block is shown as Fig.1.

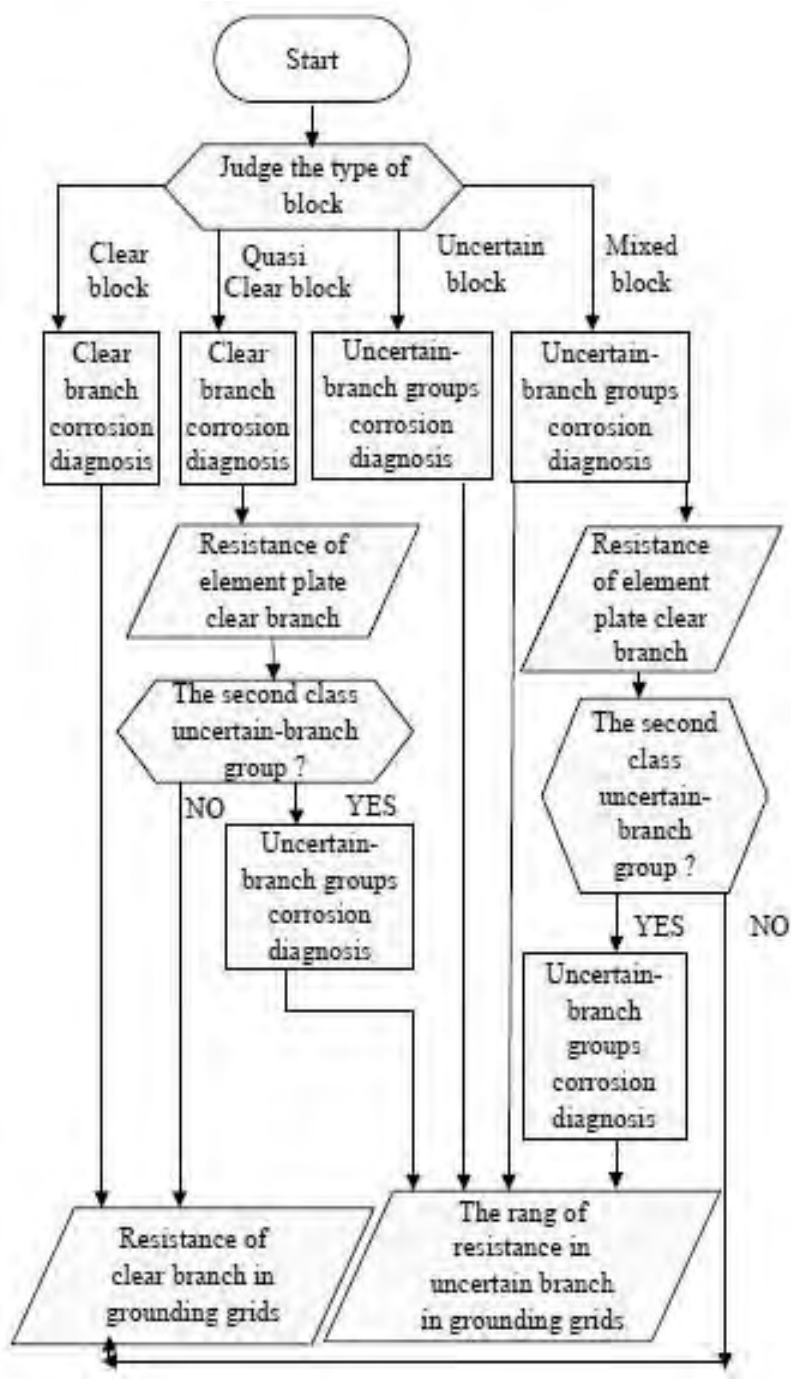

Figure 1. flow chart of diagnosis for a block

As shown in Fig.1, the corrosion diagnosis is mainly separated into two sub processes: the corrosion diagnosis of clear branches and the corrosion diagnosis of uncertain branches.

\section{2) Fully simulation test}

Based on diagnosis result of intrinsic branch which is obtained from the node oriented test result, some simulation test data is calculated for the branch resistance corrosion diagnosis in grounding grids.

A typical simulation test plan is applying constant current power excitation at both ends of intrinsic branch while calculating the voltage simulation test date between the intrinsic branches. The plan can get equations and the number of which is as same as that of intrinsic branches. Moreover, tested by superposition theorem and reciprocity theorem, the obtained equations do not repeat. Thus the 
simulation test plan is sufficient which is called typical simulation test plan.

3) Clear branch corrosion diagnosis

Two cases may appear when it refers to clear branch corrosion diagnosis in a piece of grounding grids. One is that branch resistance of a block prototype can be directly calculated by the intrinsic branch associated with, and then all will become known. The other is that resistance of a block prototype cannot be solved according to the corresponding intrinsic branch (only the result of clear branch corrosion diagnosis is out can we determine the resistance which is temporarily called unknown branch.)

a) Branch resistance in the prototypes of block known Typical simulation full test method is adopted in this case. At the ends of each branch in the prototypes direct current source is used. In light of these resistance, making use of nodes voltage equation can calculate the voltage of each branch, and the result is used as the test data of grounding grids levels (for clear blocks)or block levels(for quasi clear blocks). Then referring to an iterative least square method mentioned by [13], we can solve the branches resistance of grounding grids levels (for clear blocks)or block levels(for quasi clear blocks).

b)Branch resistance in the prototypes of block temporarily unknown.

If there are branches that resistance is temporarily unknown in the prototypes of block, we take the following steps to determine the resistance:

Step 1: At the level of grounding grids (for clear blocks) or element blocks (for quasi clear blocks) parallel branches are joined among the nodes of unknown branches in the prototype resulting in augmented blocks. Vector of $\gamma_{1}$ and $\gamma_{2}$ are paved to store the diagnosis result of branches in the augmented blocks. The resistance, corresponding to quasi branch in each branch and the resistance of which is unknown in prototypes, is considered as the initial value of each resistance of unknown branches; $n=1$.

Step 2: Half of the unknown resistance of branches in prototypes is used as the value of these resistance. Then constant current source excitation is imposed at the ends of each branch in prototype and calculates their node voltage which would become the voltage observation data of augmented blocks.

Step 3: By using the iterative least square method in [13], we estimate each branch's resistance and put the diagnosis result of branch resistance of augmented part into $\gamma_{n}$.

Step 4: If $n=2$, then we turn to step 5. If $n=n+1$, then we return to step 2 .

Step 5: If $\left\|\gamma_{1}-\gamma_{2}\right\|_{2}<\varepsilon$, then $\gamma_{2}$ is the diagnosis result of branch resistance at the level of grounding grids(for clear block) or element blocks(for quasi clear block); If not, we set $\gamma_{1}=\gamma_{2}, n=2$ and back to step 2 .

Step 6: According to circuit theory, we can calculate the resistance of each unknown branch in prototypes based on the diagnosis result of grounding grids levels (for clear blocks) or block levels (for quasi clear blocks).

4) uncertain branch corrosion diagnosis

Since branches resistance of prototypes in uncertain branch group is all clear and can be uniquely determined before grounding grids level branch corrosion diagnosis. We can adopt the method discussed in 2.3.2 part one to determine the optimal solution in iterative least square meaning of uncertain branch group. For the clear branches within, the solution is actual solution. However, for uncertain branches, referring to the dichotomy mentioned in [15], we have to make sure the possible rang of their resistance.

\section{EXAMPLES}

As shown in Fig.2, there is a grounding grid with 60 branches, serial number of nodes are indicated by number, touchable nodes are expressed as solid dots. The branch without any mark is on obstacle branch and the resistance of which is $0.1 \Omega$. The marked branches are obstacle ones and their resistance is indicated in Fig.1. With layered simplicity, each network is shown in Fig.2(d) (e). The shadow region in Fig. 2 indicates the rang of corrosion diagnosis.

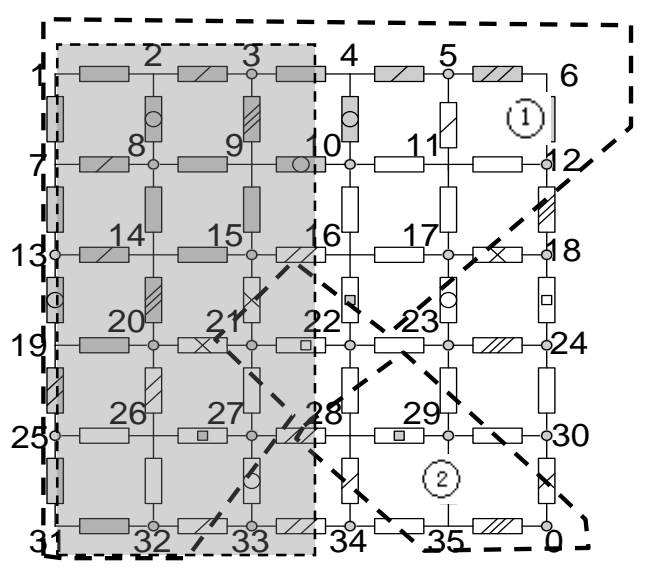

(a)example of a grounding grid with 60 branches

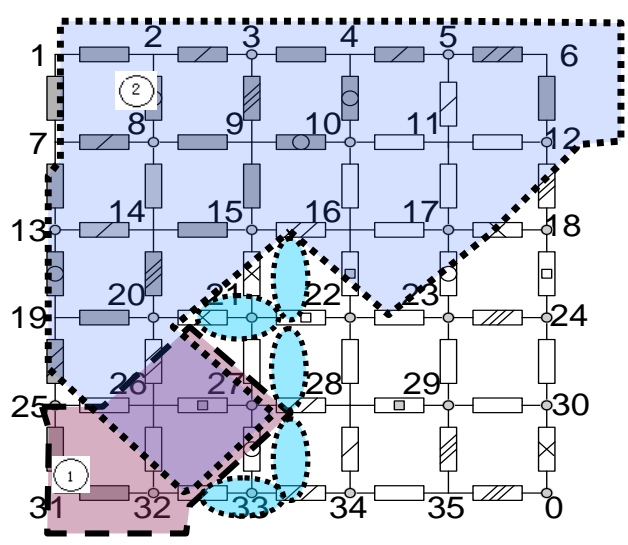

(b)seven blocks in corrosion diagnosis area 


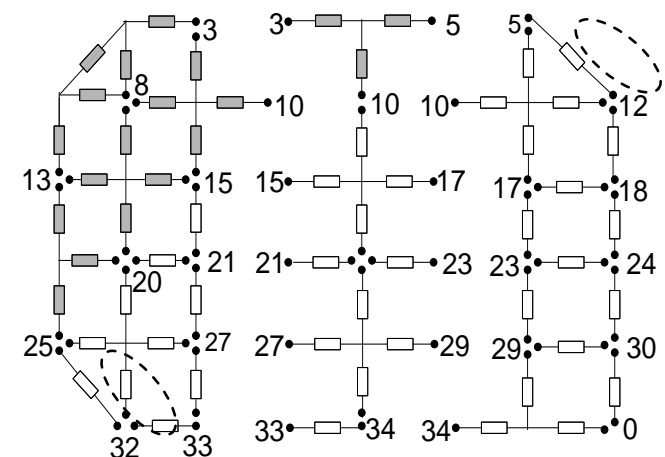

(c)element blocks

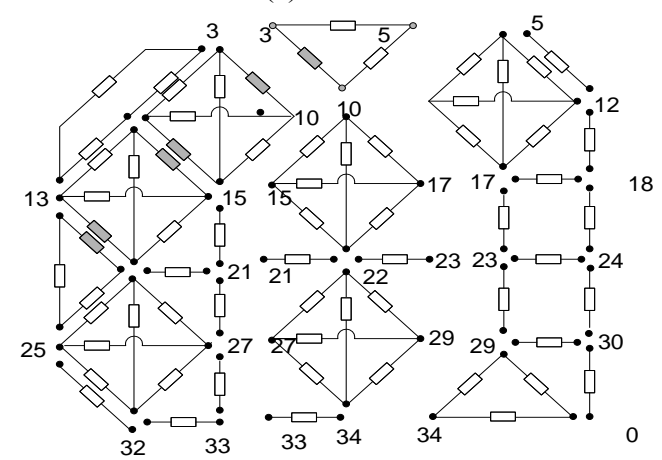

(d)element networks

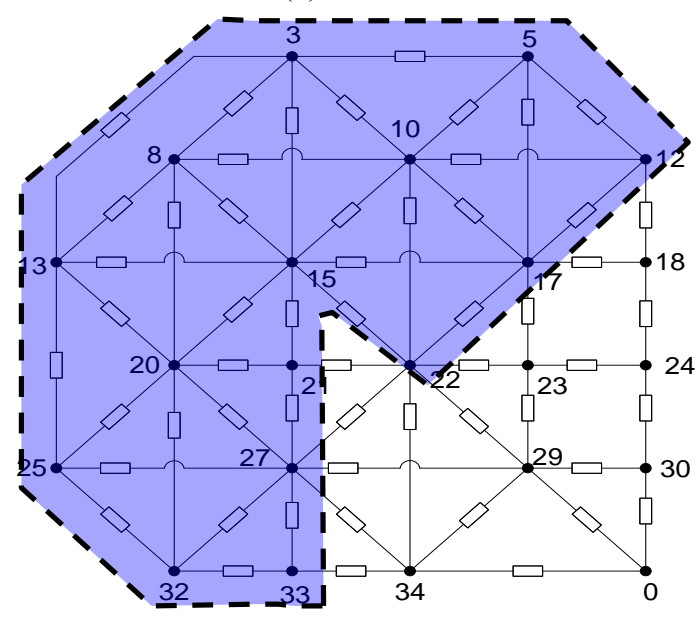

(e) intrinsic grounding grids

Figure 2. The decomposition of 60 branches grounding grids

TABLE I. Resistance of abnormal branches of grounding grid

\begin{tabular}{l|llllll}
\hline $\begin{array}{l}\text { Branch } \\
\text { type }\end{array}$ & $/$ & $/ /$ & $/ / I$ & $\square$ & O & $\times$ \\
$\begin{array}{l}\text { resistance }( \\
\mathrm{m} \Omega)\end{array}$ & 190 & 360 & 470 & 570 & 780 & $\underline{1350}$ \\
\hline
\end{tabular}

By network topology layered simplicity method, grounding grids are divided into 29 element blocks corresponding to 29 element networks. According to testability judgment analysis in [15], we can learn that the grounding grid above possesses 24 uncertain branches (as are represented in rectangular resistance frame filled by shadow), the remaining 36 branches are all clear.

Referring to 2.2, grounding grids can be divided into 19 quasi blocks, the branches contained in order are $\left\{b_{12,18}\right\} 、\left\{b_{17,18}\right\} 、\left\{b_{18,24}\right\} 、\left\{b_{17,23}\right\} 、\left\{b_{22,23}\right\}$ 、

$\left\{b_{24,23}\right\} 、\left\{b_{23,29}\right\} 、\left\{b_{24,30}\right\} 、\left\{b_{30,0}\right\} 、\left\{b_{29,30}\right\}$ 、

$\left\{b_{15,21}\right\} 、\left\{b_{20,21}\right\} 、\left\{b_{21,27}\right\} 、\left\{b_{21,22}\right\} 、\left\{b_{27,33}\right\}$ 、

$\left\{b_{32,33}\right\} 、\left\{b_{33,34}\right\} 、\{$ all the branches in dashed line

cycle (1) of Fig .2\} and \{all the branches in dashed line cycle (2) of Fig .2 3 .

In the quasi block 18 , automatic unit $\mathrm{A}$ is composed of an element network which is made up by branch $b_{20,26} 、 b_{25,26} 、 b_{26,27}$ and $b_{26,32}$. Automatic unit $\mathrm{B}$ is composed of an element network which is made up by branch $b_{10,16} 、 b_{15,16} 、 b_{16,22}$ and $b_{16,17}$. They are respectively consisting of two automatic parts in quasi blocks. The quasi block's non-automatic part $1 \#$ is constituted by element block formed by $b_{25,31}, b_{31,32}$ and automatic unit A. Non-automatic part $2 \#$ is formed by remaining non-automatic units and automatic part unit $\mathrm{A}$, B.

Division of blocks involved in corrosion diagnosis area is given in Fig.2(b). Division of uncertain branch groups referred to blocks of corrosion diagnosis area is proved in Fig.2(c). In the area of diagnosis $: b_{15,21}$ 、 $b_{20,21} 、 b_{21,27} 、 b_{27,33}$ and $b_{32,33}$ are respectively forming clear block that only contains one branch. $b_{1,2}$ 、 $b_{2,3} 、 b_{1,7} 、 b_{2,8} 、 b_{3,9} 、 b_{7,8} 、 b_{8,9} 、 b_{7,13} 、 b_{8,14}$ 、 $b_{9,15} 、 b_{13,14} 、 b_{13,19} 、 b_{14,15} 、 b_{14,20} 、 b_{19,20}$ and $b_{19,25}$ locate in non-automatic part $2 \#$ of quasi block 18 , the non-automatic part forms a mixed block in which $b_{1,2} 、 b_{2,3} 、 b_{1,7} 、 b_{2,8}$ and $b_{7,13}$ consist of uncertain branch group III, as is shown in Fig .2(c) surrounded by dashed line frame (1).

Projection of each block involved in a quasi corrosion diagnosis area is shown in Fig.2(e), sets of intrinsic branches are surrounded by dashed line. So the rang of actual diagnosis area is bigger than that of quasi corrosion diagnosis area (marked by shadow region).

Node division method presented in this paper, of course, can be also adopted to cover and solve all the intrinsic branches and get the corrosion diagnosis result. Lacking of space, we won't cover it again.

\section{V.CONCLUSION}

In view of our study above, we can come to the conclusion below:

1) The definition of blocks and their prototypes, uncertain-branch groups and their prototypes, projection of blocks and part of touchable nodes, test public nodes set and so on, make contribution to the further implementation of grounding grids corrosion diagnosis.

2) With the introduction of intrinsic branches resistance method based on division of nodes, the crossinterference is reduced and nonlinear iterative process is 
avoided. When a certain part of the grounding grid is needed to do diagnosis, only a few tests are made to meet the requirement of diagnosing.

3) A approach that based on prototypes of blocks to solve the rang of resistance of clear branches and uncertain branches is putting forward. With the proposed method, grounding grids can be obviously divided into some small scaled sub-grids to solve. As a result, the amount of computation is reduced and the efficiency is improved. An experimental grounding grid with sixty branches is used as an example. The results of corrosion diagnosis show that the proposed approaches are feasible.

\section{REFERENCE}

[1] Zhang Bo, Cui Xian g, Zhao Zhibin et al. Analysis of grounding grids at large scale substations in frequency domain [J].Proceedings of the CSEE, 2002, 22(9): 59- 63.

[2] Lu Zhiwei, Wen Xishan, Shi Yanling et al. Numerical calculation of large substation grounding grids in industrfrequency [J] .Proceedings of the CSE E, 2003, 23(12): 8993.

[3] F. P. Dawalibi, Electromagnetic fields generated by overhead and buried short conductors Part 2- Ground conductor [J]. IEEE trans. Power Delivery, 1986, 1(1) : 112- 119.

[4] Zhang Bo, Zhao Zhibin, Cui Xiang et al. Diagnosis of breaks in substation. $\mathrm{s}$ grounding grid by using the Electromagnetic method[J ] . IEE E trans. Magnetics, 2002, 38 ( 2) : 473-476.

[5] Cui Chun, Luo Xianju e, Qiu Guanyuan et al. Feed-forward mulitiple layer $\mathrm{nn}$ implementation of analog circuits fault dictionary approach[ $\mathrm{J}]$. Journal of $\mathrm{Xi}$. an Jiaotong University, 1996, 30(3) : 122- 128.
[6] Zhou Min g, He Yigang. A neuarl network approach to fault location of analog circuit with Tolerance[ $\mathrm{J}]$. Microelectronics, 2000, 30(5) : 318-320.

[7] Luo Xianjue, Qiu Guanyuan. Implementation of AC Fault dictionary by Feed-forward multilayer neuarl network [J]. Microelectronics, 1996, 26( 5) : 313-316.

[8] Hu Jun, Zhang Rong, He Jinliang et al. Novel meth od of corrosion diagnosis for grounding grid [ C ]. Proceedings of the 2000 International Conference on Power System Technology, Perth, Australia : 2000, 3: 1365-1370.

[9] Zhang Xiaoling, Huang Qingyang. Fault diagnosis of grounding grid of electric power plants and substations [ J] . Proceedings of the EPSA, 2002, 14(1): 48-51.

[10] Xiao Xinhua, Liu Hua, Chen Xianlu et al. Analysis of theory and method about the corrosion as $\mathrm{w}$ ell as the broken point of the grounding grid $[\mathrm{J}]$. Journal of Chongqing University, 2001, 24(3):72-75.

[11] Zhang Xiaoling, Chen Xianlu. The technique of the optimization applied in the grounding grid's failure diagnosis $[\mathrm{J}]$.High Voltage Engineering, 2000, 26( 4) : 64-66.

[12] Cheng Hongli, Liu Jian, Wang Sen et al. Fault diagnosis f or grounding grids based on Tabu search [ J] . High Voltage Engineering, 2007, 33(5): 139-142.

[13] Liu Jian, Wang Jianxin, Wang Sen et al. An improved algorithm of corrosion diagnosis for grounding grid \& its evaluation [J]. Proceedings of the CSEE, 2005, 25 (3): 71- 77.

[14] Huang Wenwu, Wen Xishan, Zhu Zhengguo et al .Development of the diagnosis software system for the corrosion and the broken point of the grounding grid $[\mathrm{J}]$. High Voltage Engineering, 2005, 31( 7) : 42- 44 .

[15] Liu Jian, Wang Shuqi, Li Zhizhong et al. Grounding grids corrosion diagnosis based on hierarchical simplification of network topology [J]. Proceedings of the CSEE, 2008, 28(16): $122-$ 